\title{
Plasma fibrinogen as prognostic predictor in patients with metastatic renal cell carcinoma receiving target therapy
}

\author{
Lihe Wang'" Wen Cai ${ }^{2 \#}$, Wen Kong ${ }^{2}$, Jin Zhang' ${ }^{2}$, Yonghui Chen ${ }^{2}$, Jiwei Huang ${ }^{2}$, Yiran Huang ${ }^{2}$ \\ ${ }^{1}$ Department of Urology, The Second Affiliated Hospital of Qiqihar Medical University, Qiqihar 161006, China; ${ }^{2}$ Department of Urology, Renji \\ Hospital, School of Medicine, Shanghai Jiao Tong University, Shanghai 200127, China \\ Contributions: (I) Conception and design: J Huang, Y Huang; (II) Administrative support: J Zhang, Y Chen; (III) Provision of study materials or \\ patients: L Wang, W Cai, W Kong, J Zhang, Y Chen; (IV) Collection and assembly of data: L Wang, W Cai, W Kong; (V) Data analysis and \\ interpretation: L Wang, W Cai; (VI) Manuscript writing: All authors; (VII) Final approval of manuscript: All authors. \\ "These authors contributed equally to this work. \\ Correspondence to: Yiran Huang, PhD; Jiwei Huang, PhD. Department of Urology, Renji Hospital, School of Medicine, Shanghai Jiao Tong \\ University, No.160, Pujian Road, Shanghai 200127, China. Email: yrhuangrenji@163.com; uro_renji@126.com.
}

\begin{abstract}
Background: The purpose of this work was to detect the influence of plasma fibrinogen level on the prognosis of patients with metastatic renal cell carcinoma (mRCC) before treatment of targeted therapy.

Methods: We identified mRCC patients who received first-line therapy of sorafenib or sunitinib between January 2006 to December 2016 in Renji Hospital affiliated to Shanghai Jiao Tong University School of Medicine. Kaplan-Meier method was used to estimate the progression-free survival (PFS) and overall survival (OS); log-rank test was used to compare the survival outcomes between patients with low and high fibrinogen level; and cox proportional hazard regression model was used to estimate the prognostic value. Prognostic accuracy was determined using Harrell concordance index (C-index) and receiver operating curve (ROC) analysis.
\end{abstract}

Results: Patients with high fibrinogen level both had significantly shorter median PFS (6 vs. 15 months, $\mathrm{P}<0.001)$ and $\mathrm{OS}(17$ vs. 44 months, $\mathrm{P}<0.001)$ than those with low fibrinogen level. Multivariate analysis showed that pretreatment plasma fibrinogen was an independent predictor of PFS (HR 2.873, $\mathrm{P}<0.001)$ and OS (HR 3.213, $\mathrm{P}<0.001$ ). The model built by the addition of fibrinogen improved predictive accuracy of PFS and OS compared with the International Metastatic Renal Cell Carcinoma Database Consortium (IMDC) model [Harrell C-index: 0.74 and 0.76, AUC (area under the ROC curve): 0.863, $\mathrm{P}<0.001$ ].

Conclusions: High pretreatment plasma fibrinogen could be a significant risk factor for mRCC patients receiving target therapy and increase the prognostic accuracy of established prognostic model.

Keywords: Fibrinogen; metastatic renal cell carcinoma (mRCC); prognosis; sorafenib; sunitinib

Submitted Jun 04, 2018. Accepted for publication Oct 19, 2018.

doi: $10.21037 /$ tcr.2018.10.20

View this article at: http://dx.doi.org/10.21037/tcr.2018.10.20

\section{Introduction}

Targeted therapy has brought significant changes and great benefits to metastatic renal cell carcinoma (mRCC) patients. Different from Western targeting therapy, sunitinib and sorafenib are recommend as first-line treatment by Chinese guidelines on the management of RCC (1) and these two agents are the most widely used first-line targeted drug for mRCC in China (2). The median survival time of patients with mRCC is improved, and the goal is to achieve better long-term survival (3). However, most patients with mRCC will develop to secondary drug resistance after targeted therapy of about 6 to 12 months (4). Therefore, to find pretreatment clinical markers that can effectively predict the prognosis of targeted therapies and can accurately identify patients who will benefit are important research directions for target therapy of mRCC. Although several prognostic 
models such as the International Metastatic Renal Cell Carcinoma Database Consortium (IMDC) model (5) and Memorial Sloan-Kettering Cancer Center (MSKCC) model (6) were well designed to better appropriate management decisions in patients with mRCC receiving targeted therapy, more nutritional and inflammatory markers are being added to make the traditional prognostic model more accurate recently.

Systemic inflammation is closely related to the development of the tumor (7). There are many clinical hematological indicators which could reflect body inflammation levels. Routine laboratory inflammation indexes such as CRP (8), albumin (9) were proved to be important prognostic indicators for mRCC patients receiving target therapy. Recently, some new combined indexes like neutrophil to lymphocyte ratio (NLR) (10), platelet to lymphocyte ratio (PLR) $(11,12)$, prognostic nutritional index (PNI) (13) were also found to be effective prognostic biomarkers. Fibrinogen is a kind of glycoprotein which is synthesized and secreted by the liver, and it is a common and important blood coagulation test index in clinic. Similarly, fibrinogen is also highly expressed in different tumors and closely related to tumor activity (14). High fibrinogen level was proved to be a risk factor in non-mRCC (15) and localized upper tract urothelial carcinoma (16). And for mRCC patients treated by interleukin-2, high fibrinogen levels were also found to be independently associated with the tumor responses and survival outcomes (17). However, the prognostic evidence of fibrinogen in mRCC patients treated with targeted therapy remains elusive.

Thus, in this study, we conducted a large retrospective study to analyze the prognostic role of fibrinogen in metastatic RCC patients receiving target therapy and added fibrinogen to IMDC model to prove its prognostic efficiency.

\section{Methods}

\section{Study design and population}

The electronic medical records and laboratory data of mRCC patients from March 2006 to December 2016 at the Department of Urology in Renji Hospital affiliated to Shanghai Jiao Tong University School of Medicine were analyzed retrospectively. Ethical ratification was authorized by the clinical research ethics committee of Renji Hospital. This study involved patients who was confirmed as renal cell cancer with metastasis histologically or cytologically and received first-line targeted treatment of sunitinib or sorafenib. The blood routine, liver and kidney function and fibrinogen of whom were recorded within one to two weeks before targeted treatment. Patients who did not conform to the above criteria and who with incomplete clinical medical records or follow-up data were excluded from this study.

\section{Clinical and pathological evaluation}

We retrieved the patient's clinical pathology and examination information from the medical records database. Pretreatment baseline assessment was performed before target treatment began, including hematology and radiographic examination. Fuhrman nuclear grade and clinical staging based on the AJCC 2010 TNM classification. IMDC focuses on 6 principal adverse prognostic factors: Karnofsky performance status (KPS) score less than 80; neutrophilia; low hemoglobin; elevated corrected calcium; thrombocytosis; and the time from diagnosis to treatment less than 1 year. Zero risk factor belongs to favorable IMDC group; 1-2 risk factors belong to intermediate IMDC group and 3-6 belong to poor group (5).

\section{Treatment and follow up}

Sorafenib was taken $400 \mathrm{mg}$ BID orally in cycles of 4 weeks and sunitinib was taken $50 \mathrm{mg}$ QD orally for the first 4 weeks in a 6-week cycle (4-week on, 2-week off-4/2 schedule). Dose adjustments, including dose increase or dose reduction will be based on adverse events experienced by the individual patient. After the target treatment began, the patients were followed up monthly and the imaging examination was reviewed every $2-3$ months until the patient was lost to follow up or died. Progressionfree survival (PFS) was defined as the time from onset of target therapy to first documentation of objective tumor progression or the last visiting day recorded if the disease did not progress. overall survival (OS) was defined as the time from date of onset of target therapy to date of death due to any cause or the last visiting day recorded if the patients were alive.

\section{Statistical analyses}

All analyses were performed using the SPSS version 22.0 (IBM Corp., Armonk, NY, USA) and R (R version 3.4.3). Pearson's Chi-squared test was used to compared categorical 
variables. Receiver operating curve (ROC) analysis was used to determine the best cutoff point of plasma fibrinogen levels to separate patients' survival outcomes. The optimal threshold of fibrinogen value was determined on the ROC curve with a maximum Youden index. Kaplan-Meier method was used to describe survival curve and log-rank test was used to compare the PFS and OS. Cox regression analysis and Cox's proportional hazard model was used to calculate prognostic value of different variables. C-index and ROC analysis were used to estimate predictive ability of survival outcomes. All statistical tests were two-sided, and the level of significant $\mathrm{P}$ was $<0.05$. The $\mathrm{C}$-index was built based on training set with the R package "survival".

\section{Results}

\section{Clinical characteristics}

A total of 318 cases of mRCC who treated with targeted therapy were retrospectively retrieved from medical records. Of these patients, 164 patients were complied with our inclusion criteria and included in this study. The clinical characteristics were shown in Table 1. ROC analysis to determine the cutoff point of fibrinogen was shown in Figure 1 and the best cutoff value of fibrinogen was $2.940 \mathrm{~g} / \mathrm{L}$. Ninety-four patients constituted low fibrinogen group $(<2.940 \mathrm{~g} / \mathrm{L})$ and 70 patients constituted high fibrinogen $(\geq 2.940 \mathrm{~g} / \mathrm{L})$ groups. Our final cohort included 122 men (74.4\%) and 42 women (25.6\%). Median age was 60 years (24-82 years). Of them, 7 patients Furman grade could not be found in the database. Seventy-four (45.1\%) patients belonged favorable IMDC group, 64 (39.0\%) belonged intermediate IMDC group and 26 (15.9\%) belonged poor IMDC group. Sorafenib was administered as first-line therapy in $101(61.6 \%)$ patients whereas sunitinib was $63(38.4 \%)$.

\section{Correlation between fibrinogen and clinical and pathological characteristics}

The median (range) pretreatment plasma fibrinogen level was $2.76(1.98-4.56) \mathrm{g} / \mathrm{L}$. No significant difference was found in sex, age, histology, prior cytokines therapy or nephrectomy, Fuhrman grade and the kind of first-line targeted agents between two groups. In the high fibrinogen level group, there were more patients with $\geq 2$ metastasis sites $(38.6 \%$ vs. $23.4 \%, \mathrm{P}=0.036)$ and more patients with poorer IMDC risk grade $(\mathrm{P}=0.003)$.

\section{Correlation between pretreatment fibrinogen and survival outcomes}

The median follow-up period was 26 months. The overall median PFS of all 164 patients was 10 months (95\% CI, 8-12 months), and the median OS was and 28 months (95\% CI, 23-33 months). One-year survival rate was $71.3 \%$ and 2-year survival rate was $45.1 \%$. As shown in Figure 2, median PFS (6 vs. 15 months, $\mathrm{P}<0.001)$ and OS $(17$ vs. 44 months, $\mathrm{P}<0.001)$ in the high plasma fibrinogen group were significantly shorter than low plasma fibrinogen group. We further compared the survival outcomes between high and low plasma fibrinogen level groups in different IMDC risk patients in Figure 3. In the sub-group of favorable, intermediate or poor IMDC, the median PFS and OS were also significant shorter in high plasma fibrinogen group than low.

The prognostic value of diffident clinical pathological parameters on PFS and OS was shown in Tables 2 and 3. Multivariate analysis revealed that Fuhrman grade, number of metastasis sites, IMDC were significant predictor of PFS and OS. We also identified that plasma fibrinogen $(<2.940$ and $\geq 2.940 \mathrm{~g} / \mathrm{L}$ ) remained a significant predictor of shorter OS (HR 3.213, 95\% CI, 2.138-4.827, $\mathrm{P}<0.001$ ) and PFS (HR 2.873, $95 \%$ CI, 2.002-4.123, $\mathrm{P}<0.001)$ in the multivariate analysis. Further, when fibrinogen was analyzed as continuous variable, plasma fibrinogen was remaining as a significant predictor for PFS (HR 2.035, 95\% CI, 1.519-2.726, $\mathrm{P}<0.001$ ) and OS (HR $1.968,95 \%$ CI, 1.475-2.627, $\mathrm{P}<0.001$ ) (data not shown).

\section{Predictive accuracy of pretreatment fibrinogen and IMDC}

C-index was presented in Table 4. The C-index of fibrinogen $(<2.940$ and $\geq 2.940 \mathrm{~g} / \mathrm{L})$ for PFS and OS was 0.65 (95\% CI, $0.60-0.70)$ and 0.66 (95\% CI, 0.61-0.71). After the adding fibrinogen to the base IMDC model, C-index for PFS was improved from 0.68 of IMDC model to 0.74 , and for OS, it was improved from 0.69 to 0.76 . The ROC curve of the IMDC and combined model to predict the prognosis was plotted in Figure 4. The AUC was also improved form the 0.793 (95\% CI, 0.722-0.852) of IMDC to 0.863 (95\% CI, $0.801-0.921, \mathrm{P}<0.001)$ after the addition of fibrinogen level.

\section{Discussion}

Several established prognostic models are used to predict the clinical outcomes of mRCC. According to the IMDC model, pretreatment anemia, thrombocytosis, neutrophilia, KPS and time from diagnosis to first-line targeted therapy 
Table 1 Patient baseline and clinicopathological characteristics

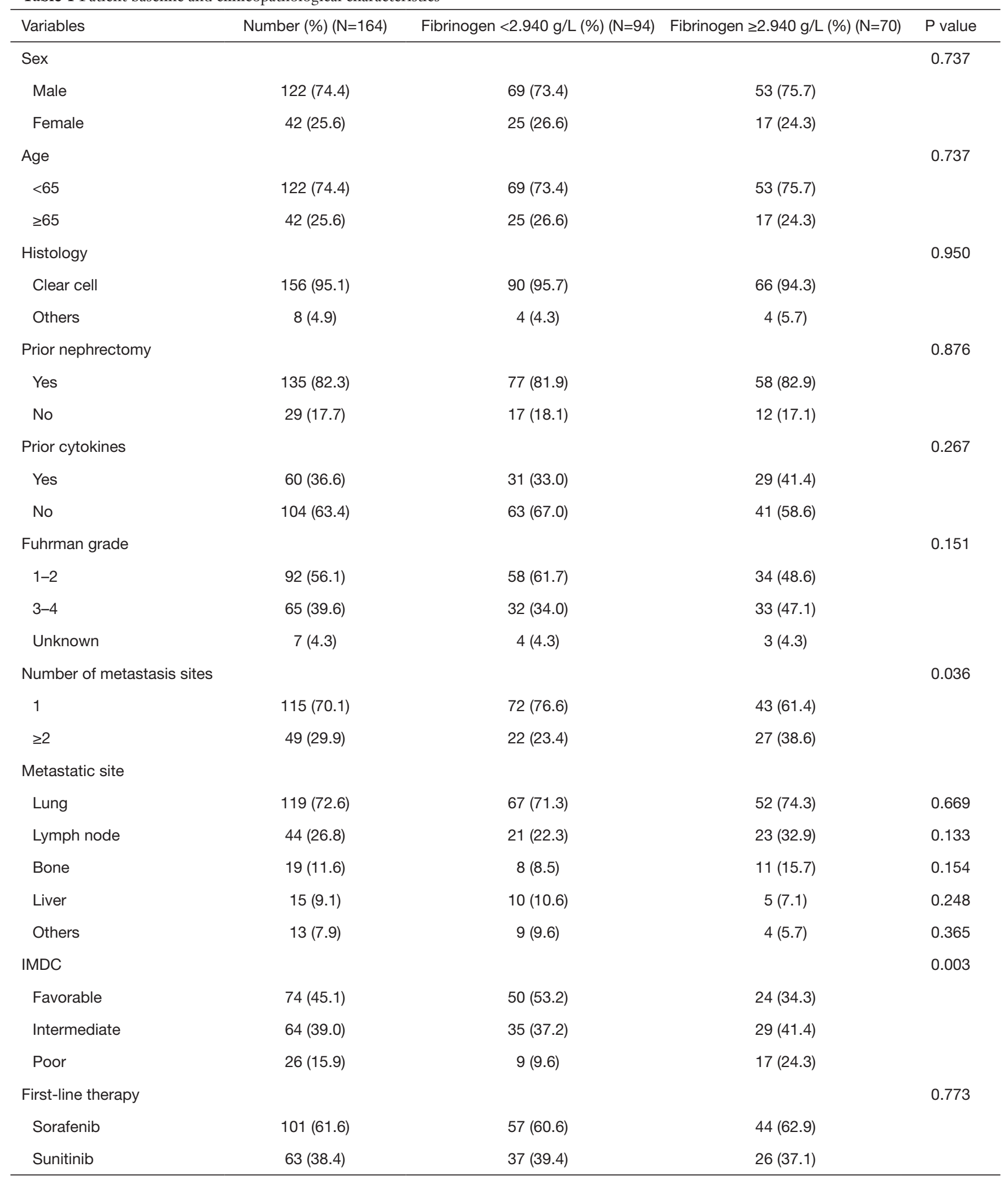

IMDC, International Metastatic Renal Cell Carcinoma Database Consortium model. 
are prognostic factors for mRCC. Moreover, system inflammation markers like CRP (8), NLR (10) an PLR (11) represent new independent survival outcomes predictors of mRCC patients. Fibrinogen was also proved to be a prominent prognostic indicator in different cancers such as hepatocellular carcinoma (18), esophageal squamous cell carcinoma (19) and localized renal cell carcinoma (20). Tsimafeyeu et al. (17) have compared OS time between two groups of mRCC patients with and without hypercoagulability then found that hypercoagulability was an independent risk factor for mRCC. However, they have not analyzed the fibrinogen level separately. The number of patients was limited in their study and they have not specifically included patients who receive targeted therapy. Therefore, the potential prognostic significance of fibrinogen is still limited in target treatment of mRCC. New biomarker for prognosis is important

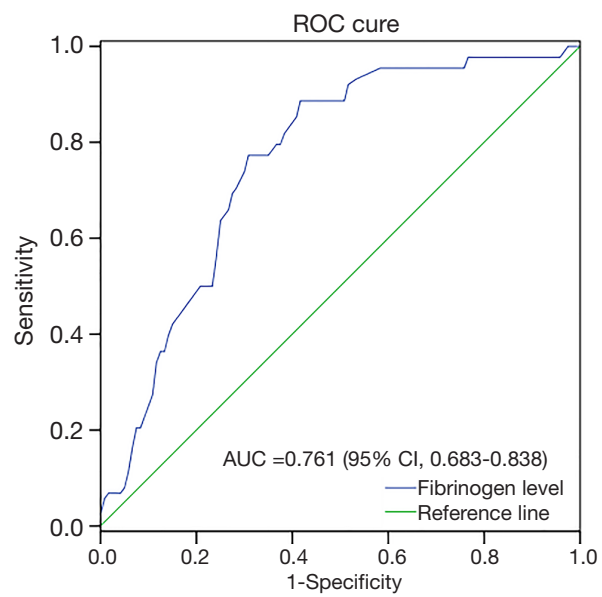

Figure 1 Receiver operating characteristic (ROC) analysis of optimal fibrinogen cutoff level. since they would guide clinical judgment and treatment for individual patient. To the best of our knowledge, this work is the first to demonstrate the prognostic value of pretreatment plasma fibrinogen level in mRCC patients receiving target therapy. Finally, we found that fibrinogen could accurately predict the clinical outcomes of $\mathrm{mRCC}$ and increase the accuracy of prognostic prediction of IMDC model.

Systemic inflammation is an important risk factor for tumor progression. The change of plasma fibrinogen is associated with abnormal coagulation and inflammation of the body (20). Renal cell carcinoma cells may activate the coagulation cascade, and thus increase the plasma fibrinogen level and promote the hematogenous metastasis (20). Fibrinogen can be synthesized endogenously through the tumor cell and it can regulate the growth of cancer cells by binding to VEGF, FGF-2 and PDGF (21). In addition, VEGF can also conversely stimulate the endothelial cells, activate the coagulation cascade, and increase the fibrinogen level (22). Blocking the VEGF and PDGF signaling pathways may reverse the physiological processes of $V H L$ gene function and thus inhibit tumor growth, which is the rationale for anti-angiogenic therapy for mRCC (23). Thus, on one hand, high plasma fibrinogen might be related to the progress of renal cancer, and on the other hand it is related to drug resistance of target therapy.

As to clinic-pathological parameters, the higher clinical stages of multiple tumors were reported to be accompanied with an increased plasma fibrinogen level (24-26). Du et al. (27) found that a higher plasma fibrinogen level was significantly correlated to higher Fuhrman grade, pathologic $\mathrm{T}$ stage and larger tumor diameter in non-mRCC. Further, Pichler et al. (15) and Obata et al. (20) found it also predicts worse survival endpoints in localized renal cell carcinoma. In
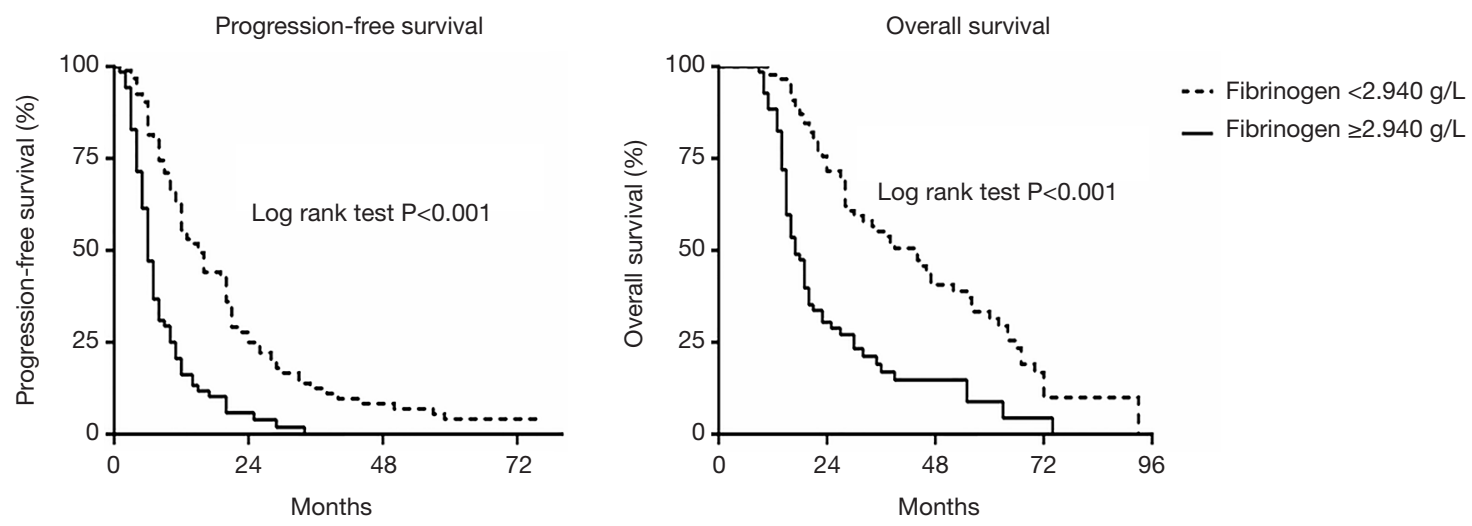

Figure 2 Progression-free survival (PFS) and overall survival (OS) of patients with high plasma fibrinogen vs. low high plasma fibrinogen. 

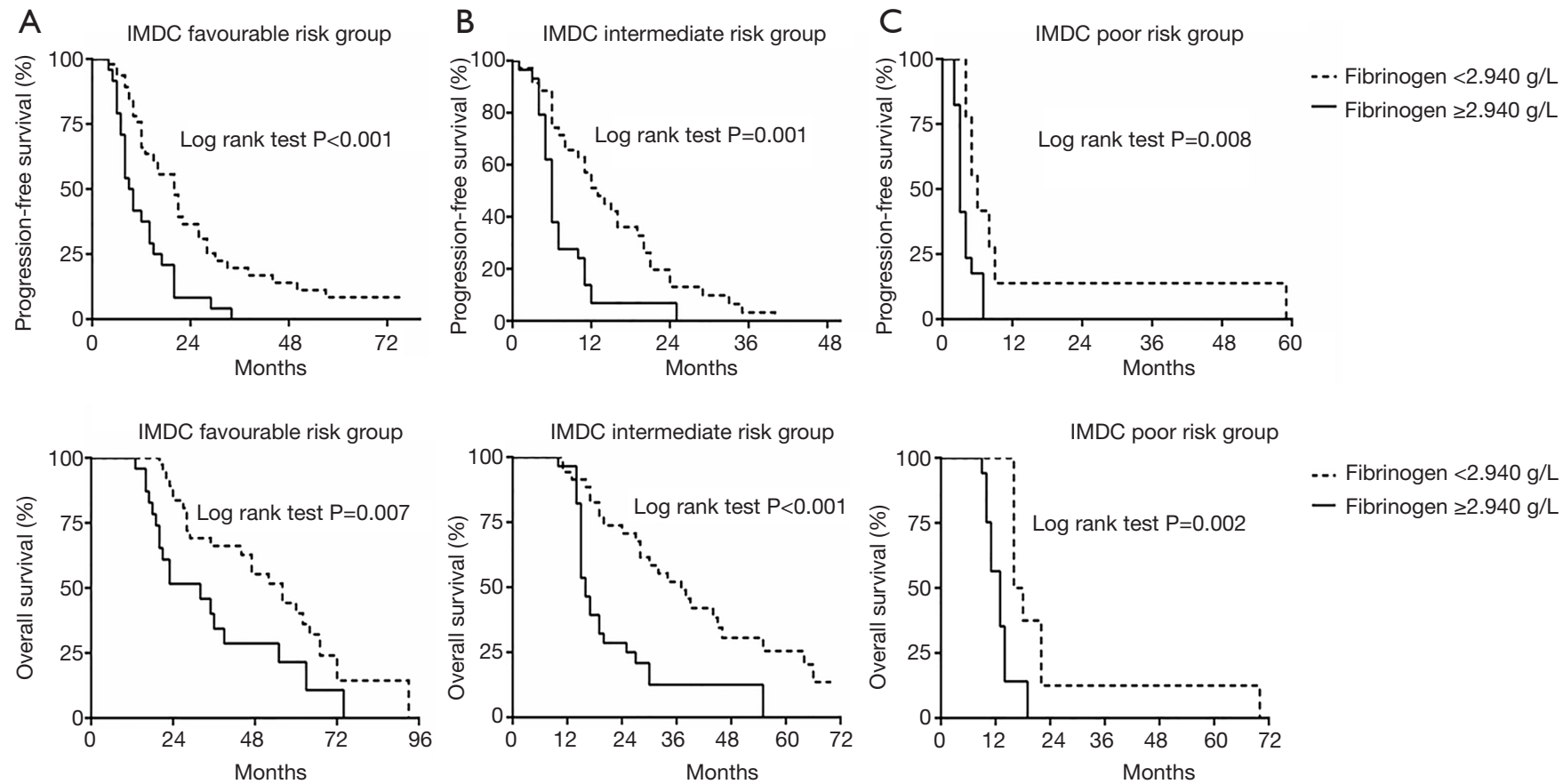

Figure 3 Kaplan-Meier survival curves stratified by IMDC risk model. (A) Favorable risk; (B) intermediate risk; (C) poor risk. IMDC, International Metastatic Renal Cell Carcinoma Database Consortium model.

Table 2 Univariable and multivariable Cox regression models to predict PFS

\begin{tabular}{|c|c|c|c|c|}
\hline Variables & \multicolumn{2}{|c|}{ Univariable } & \multicolumn{2}{|c|}{ Stepwise analysis } \\
\hline Sex (male versus female) & $0.999(0.689-1.450)$ & 0.998 & - & - \\
\hline Age ( $\geq 65$ vs. <65) & $1.016(0.699-1.476)$ & 0.934 & - & - \\
\hline Pathology (nccRCC vs. ccRCC) & $1.714(0.745-3.943)$ & 0.205 & - & - \\
\hline History of cytokines (no vs. yes) & $1.308(0.927-1.845)$ & 0.126 & - & - \\
\hline Fuhrman grade & & 0.003 & & 0.003 \\
\hline $1-2$ & Ref & - & Ref & - \\
\hline $3-4$ & $1.662(1.187-2.328)$ & 0.003 & 1.747 (1.229-2.481) & 0.002 \\
\hline Heng risk grade & & $<0.001$ & & $<0.001$ \\
\hline Favourable & Ref & - & Ref & - \\
\hline Intermediate & $1.796(1.252-2.576)$ & 0.001 & $1.740(1.202-2.518)$ & 0.003 \\
\hline Poor & $4.183(2.554-6.850)$ & $<0.001$ & $4.873(2.967-8.390)$ & $<0.001$ \\
\hline Fibrinogen ( $\geq 2.940$ vs. $<2.940 \mathrm{~g} / \mathrm{L})$ & $2.754(1.949-3.892)$ & $<0.001$ & $2.873(2.002-4.123)$ & $<0.001$ \\
\hline Drug category (sunitinib vs. sorafenib) & $1.056(0.755-1.476)$ & 0.751 & - & - \\
\hline
\end{tabular}

PFS, progression-free survival; ccRCC, clear cell renal cell carcinoma; nccRCC, non-clear cell renal cell carcinoma. 
Table 3 Univariable and multivariable Cox regression models to predict OS

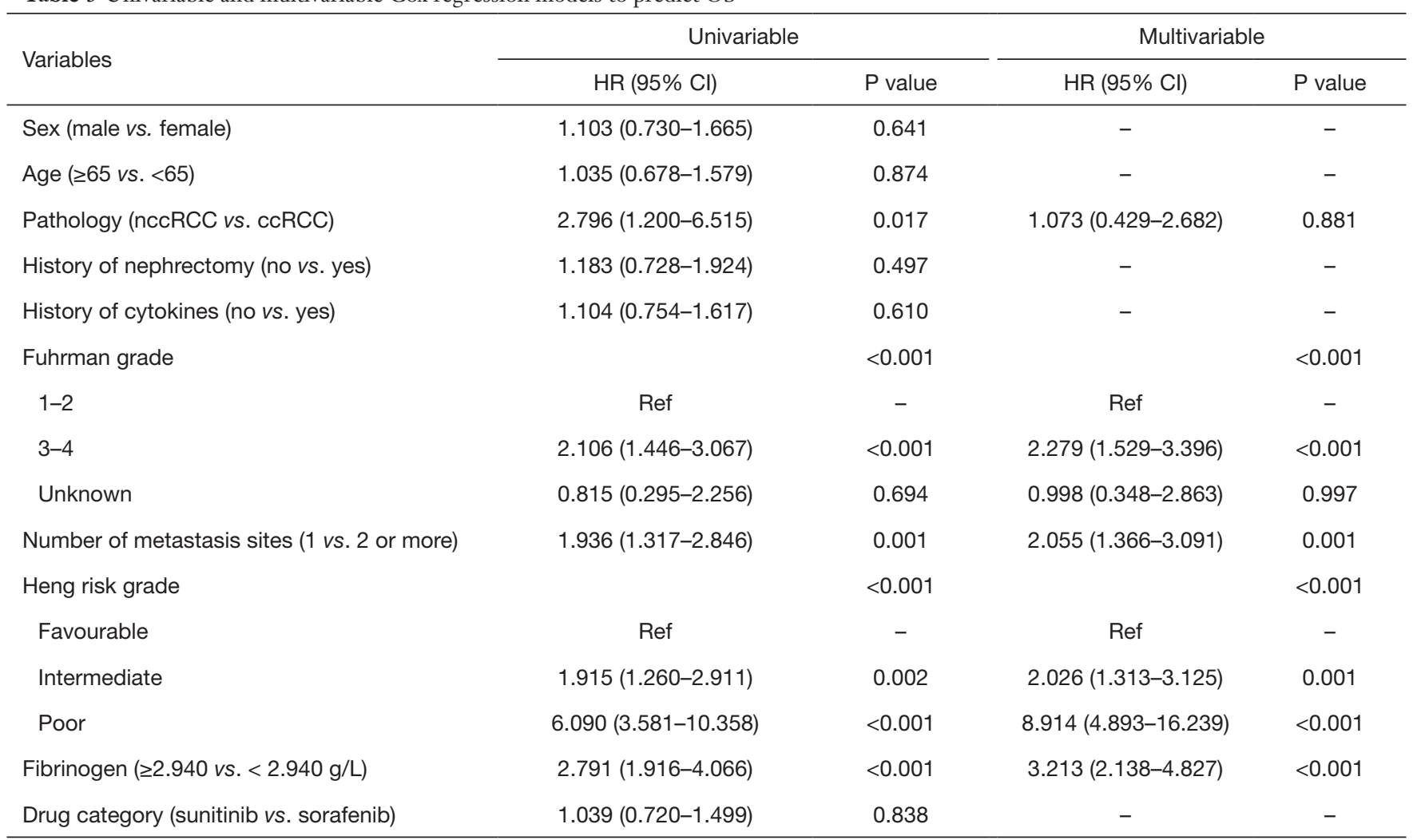

OS, overall survival; ccRCC, clear cell renal cell carcinoma; nccRCC, non-clear cell renal cell carcinoma.

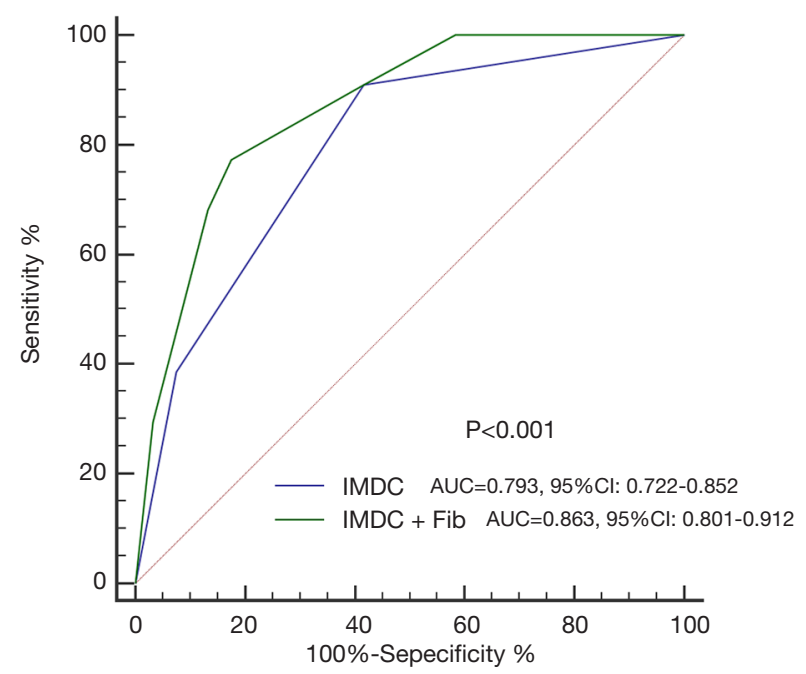

Figure 4 Receiver operating characteristic (ROC) analysis to predict survival: plasma fibrinogen + IMDC vs. IMDC. IMDC, International Metastatic Renal Cell Carcinoma Database Consortium model.
Table 4 C-index of IMDC and IMDC with fibrinogen

\begin{tabular}{lcc}
\hline \multirow{2}{*}{ Variables } & \multicolumn{2}{c}{ C-index (std) } \\
\cline { 2 - 3 } IMDC & $0.68(0.027)$ & $0.69(0.028)$ \\
Fibrinogen & $0.65(0.025)$ & $0.66(0.026)$ \\
IMDC + fibrinogen & $0.74(0.029)$ & $0.76(0.031)$ \\
\hline IMDC, International Metastatic Renal Cell Carcinoma Database \\
$\begin{array}{l}\text { Consortium model; PNI, prognostic nutritional index; PFS, } \\
\text { progression-free survival; OS, overall survival. }\end{array}$
\end{tabular}

mRCC, we also found that the proportion of patients with more metastatic sites in the high fibrinogen level group was higher than the low group $(\mathrm{P}=0.036)$. Clinical studies suggest that hypercoagulability is common in the blood of patients with malignant tumors and it can accelerate the metastasis of tumor then ultimately affect the prognosis of patients (14), which was consistent with our research. More metastatic sites might mean a higher plasma fibrinogen level. We also found that there were more patients with poorer IMDC risk patients 
in high fibrinogen group. In order to better compare the fibrinogen level with the basic IMDC model and eliminate this interference, we separately analyzed the survival curves of the high and low fibrinogen level in each subgroup of IMDC. The results showed that both the PFS and the OS in the high plasma fibrinogen level group were significantly lower than the low fibrinogen group whether in favorable, intermediate or poor risk IMDC group. Similarly, in multifactor analysis, except other interfering factors, fibrinogen remained an independent risk factor for survival outcomes.

At present, the commonly used prognostic models like IMDC show good accuracy and applicability in clinical application of targeted therapy in mRCC (4). With further research on the mechanism of inflammation, tumor occurrence and development, and new-found prognostic factors of mRCC, more accurate and adaptable prognosis models for target treatment of mRCC are expected to be developed and applied. In this work, we found that adding fibrinogen to base model was able to raise the predictive accuracy. The C-index of IMDC for FPS and OS was 0.68 and 0.69 , and the AUC was 0.793 . After adding fibrinogen to it, predictive accuracy increased to 0.74 and 0.76 . AUC could also be improved to 0.863 . Fibrinogen is easy to assess and could be routinely measured before target therapy. Addition of fibrinogen can help predict the prognosis of clinical more accurately, and further to help scientifically develop the next target treatment strategy.

This study had several limitations. Firstly, this is a singlecenter retrospective study. Despite we have analyzed the data which were strictly included according to criteria, some confounding and bias were still inevitable. Secondly, different patients would choose different second- or third-line treatment which might have significant inference on OS time. Thus, Future researches are recommended to study the prognostic role of plasma fibrinogen level, reveal its underlying mechanisms and improve predictive accuracy of traditional model in order to a better guide for targeted therapy in mRCC.

\section{Conclusions}

This study showed basal plasma fibrinogen status could be used as an independent prognostic parameter for the PFS and OS of patients with mRCC receiving target therapy. It could also be an important complement to the traditional prognostic model. Monitoring the fibrinogen status is not only an effective measure to detect coagulation status, but also play an important role in controlling the development and predicting prognosis of target treatment in mRCC.

\section{Acknowledgments}

Funding: This study was supported by grant number SFGG201442 from Qiqihar Science and Technology Plan Project, 81472378 from National Natural Science Foundation of China, PYXJS16-008 from the Incubating Program for Clinical Research and Innovation of Renji Hospital and 16CR3062B from the Shen Kang - 3 years action plan program.

\section{Footnote}

Conflicts of Interest: All authors have completed the ICMJE uniform disclosure form (available at http://dx.doi. org/10.21037/tcr.2018.10.20). The authors have no conflicts of interest to declare.

Ethical Statement: The authors are accountable for all aspects of the work in ensuring that questions related to the accuracy or integrity of any part of the work are appropriately investigated and resolved. Ethical ratification was authorized by the clinical research ethics committee of Renji Hospital (2005-RJ006). All procedures performed in studies involving human participants were in accordance with the ethical standards of the Renji Hospital and with the 1964 Helsinki Declaration and its later amendments or comparable ethical standards. The written informed consent was waived due to the retrospective nature of the study.

Open Access Statement: This is an Open Access article distributed in accordance with the Creative Commons Attribution-NonCommercial-NoDerivs 4.0 International License (CC BY-NC-ND 4.0), which permits the noncommercial replication and distribution of the article with the strict proviso that no changes or edits are made and the original work is properly cited (including links to both the formal publication through the relevant DOI and the license). See: https://creativecommons.org/licenses/by-nc-nd/4.0/.

\section{References}

1. Guo J, Ma J, Sun Y, et al. Chinese guidelines on the management of renal cell carcinoma (2015 edition). Ann Transl Med 2015;3:279.

2. Ye DW, Zhang HL. Critical appraisal of sorafenib in the treatment of Chinese patients with renal cell carcinoma. Onco Targets Ther 2014;7:925-35.

3. Hsieh JJ, Purdue MP, Signoretti S, et al. Renal cell carcinoma. Nat Rev Dis Primers 2017;3:17009. 
4. Harshman LC, Xie W, Bjarnason GA, et al. Conditional survival of patients with metastatic renal-cell carcinoma treated with VEGF-targeted therapy: a population-based study. Lancet Oncol 2012;13:927-35.

5. Ko JJ, Xie W, Kroeger N, et al. The International Metastatic Renal Cell Carcinoma Database Consortium model as a prognostic tool in patients with metastatic renal cell carcinoma previously treated with first-line targeted therapy: a population-based study. Lancet Oncol 2015;16:293-300.

6. Motzer RJ, BacikJ, Murphy BA, et al. Interferon-alfa as a comparative treatment for clinical trials of new therapies against advanced renal cell carcinoma. J Clin Oncol 2002;20:289-96.

7. Coussens LM, Werb Z. Inflammation and cancer. Nature 2002;420:860-7.

8. Teishima J, Kobatake K, Kitano H, et al. The impact of change in serum C-reactive protein level on the prediction of effects of molecular targeted therapy in patients with metastatic renal cell carcinoma. BJU Int 2016;117:E67-74.

9. Stenman M, Laurell A, Lindskog M. Prognostic significance of serum albumin in patients with metastatic renal cell carcinoma. Med Oncol 2014;31:841.

10. Templeton AJ, Knox JJ, Lin X, et al. Change in Neutrophilto-lymphocyte Ratio in Response to Targeted Therapy for Metastatic Renal Cell Carcinoma as a Prognosticator and Biomarker of Efficacy. Eur Urol 2016;70:358-64.

11. Gunduz S, Mutlu H, Tural D, et al. Platelet to lymphocyte ratio as a new prognostic for patients with metastatic renal cell cancer. Asia Pac J Clin Oncol 2015;11:288-92.

12. Koo KC, Lee KS, Cho KS, et al. Comprehensive analysis and validation of contemporary survival prognosticators in Korean patients with metastatic renal cell carcinoma treated with targeted therapy: prognostic impact of pretreatment neutrophilto-lymphocyte ratio. Int Urol Nephrol 2016;48:985-92.

13. Cai W, Zhong H, Kong W, et al. Significance of preoperative prognostic nutrition index as prognostic predictors in patients with metastatic renal cell carcinoma with tyrosine kinase inhibitors as first-line target therapy. Int Urol Nephrol 2017;49:1955-63.

14. Sahni A, Simpson-Haidaris PJ, Sahni SK, et al. Fibrinogen synthesized by cancer cells augments the proliferative effect of fibroblast growth factor-2 (FGF-2). J Thromb Haemost 2008;6:176-83.

15. Pichler M, Hutterer GC, Stojakovic T, et al. High plasma fibrinogen level represents an independent negative prognostic factor regarding cancer-specific, metastasis-free, as well as overall survival in a European cohort of non-metastatic renal cell carcinoma patients. Br J Cancer 2013;109:1123-9.

16. Pichler M, Dalpiaz O, Ehrlich GC, et al. Validation of the preoperative plasma fibrinogen level as a prognostic factor in a European cohort of patients with localized upper tract urothelial carcinoma. J Urol 2014;191:920-5.

17. Tsimafeyeu IV, Demidov LV, Madzhuga AV, et al. Hypercoagulability as a prognostic factor for survival in patients with metastatic renal cell carcinoma. J Exp Clin Cancer Res 2009;28:30.

18. Gan W, Yi Y, Fu Y, et al. Fibrinogen and C-reactive protein score is a prognostic index for patients with hepatocellular carcinoma undergoing curative resection: a prognostic nomogram study. J Cancer 2018;9:148-56.

19. Kijima T, Arigami T, Uchikado Y, et al. Combined fibrinogen and neutrophil-lymphocyte ratio as a prognostic marker of advanced esophageal squamous cell carcinoma. Cancer Science 2017;108:193-9.

20. Obata J, Tanaka N, Mizuno R, et al. Plasma fibrinogen level: an independent prognostic factor for diseasefree survival and cancer-specific survival in patients with localised renal cell carcinoma. BJU Int 2016;118:598-603.

21. Witsch E, Sela M, Yarden Y. Roles for growth factors in cancer progression. Physiology (Bethesda) 2010;25:85-101.

22. Verheul HM, van Erp K, Homs MY, et al. The relationship of vascular endothelial growth factor and coagulation factor (fibrin and fibrinogen) expression in clear cell renal cell carcinoma. Urology 2010;75:608-14.

23. Versteeg HH, Peppelenbosch MP, Spek CA. Tissue factor signal transduction in angiogenesis. Carcinogenesis 2003;24:1009-13.

24. Mei Y, Zhao S, Lu X, et al. Clinical and Prognostic Significance of Preoperative Plasma Fibrinogen Levels in Patients with Operable Breast Cancer. PLoS One 2016;11:e0146233.

25. Troppan KT, Melchardt T, Wenzl K, et al. The clinical significance of fibrinogen plasma levels in patients with diffuse large B cell lymphoma. J Clin Pathol 2016;69:326-30.

26. Zhu LR, Li J, Chen P, et al. Clinical significance of plasma fibrinogen and D-dimer in predicting the chemotherapy efficacy and prognosis for small cell lung cancer patients. Clin Transl Oncol 2016;18:178-88.

27. Du J, Zheng JH, Chen XS, et al. High preoperative plasma fibrinogen is an independent predictor of distant metastasis and poor prognosis in renal cell carcinoma. Int $\mathrm{J}$ Clin Oncol 2013;18:517-23.

Cite this article as: Wang L, Cai W, Kong W, Zhang J, Chen Y, Huang J, Huang Y. Plasma fibrinogen as prognostic predictor in patients with metastatic renal cell carcinoma receiving target therapy. Transl Cancer Res 2018;7(6):1384-1392. doi: 10.21037/ tcr.2018.10.20 